\title{
Long-Distance Ionic Diffusion in Cesium Lead Mixed Halide Perovskite Induced by Focused-Illumination
}

Weijian Chen, ${ }^{1}$ Wei Li, ${ }^{2,3,4}$ Zhixing Gan, ${ }^{1,5}$ Yi-Bing Cheng, ${ }^{2,6}$ Baohua Jia, ${ }^{1 *}$ Xiaoming Wen ${ }^{1 *}$

1, Centre for Translational Atomaterials, Swinburne University of Technology, Hawthorn 3122, Australia

2, State Key Laboratory of Advanced Technology for Materials Synthesis and Processing, Wuhan University of Technology, Wuhan, 430070, China

3, Department of Chemical Engineering, Monash University, Victoria 3800, Australia

4, ARC Centre of Excellence in Exciton Science, Monash University, Victoria 3800, Australia.

5, Key Laboratory of Optoelectronic Technology of Jiangsu Province, School of Physics and Technology, Nanjing Normal University, Nanjing 210023, China

6, Department of Materials Science and Engineering, Monash University, Victoria 3800, Australia.

(A)

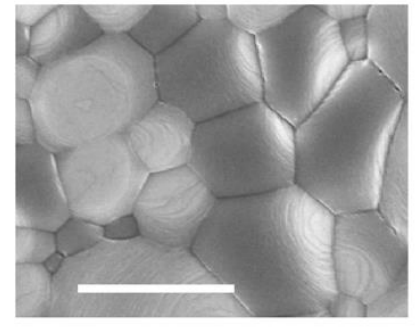

(D)

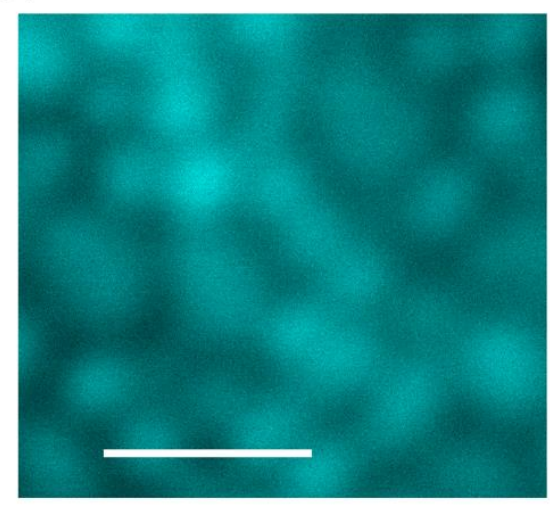

(B)

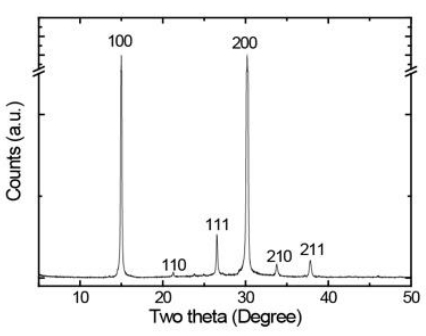

(E)

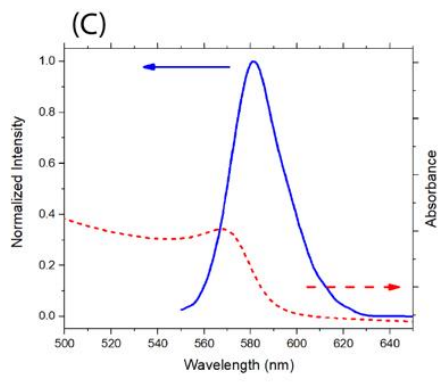

(E)

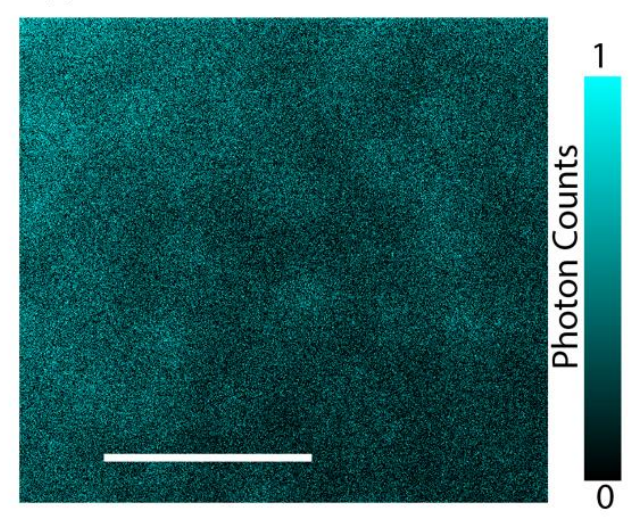

Figure S1. Pristine properties of CsPbIBr2 film. (A) SEM image of the CsPbIBr 2 thin film; (B) X-ray diffraction patterns for the $\mathrm{CsPbIBr}_{2}$ film; (C) PL \& absorbance spectra of the unexposedCsPbIBr $\mathrm{Cilm}_{2}$. PL scanning image of (D) Br-phase (580nm-610nm) and (E) I-phase (630 nm-690 nm) of the unexposed $\mathrm{CsPbIBr}_{2}$ film; all scale bars are $1 \mu \mathrm{m}$. 

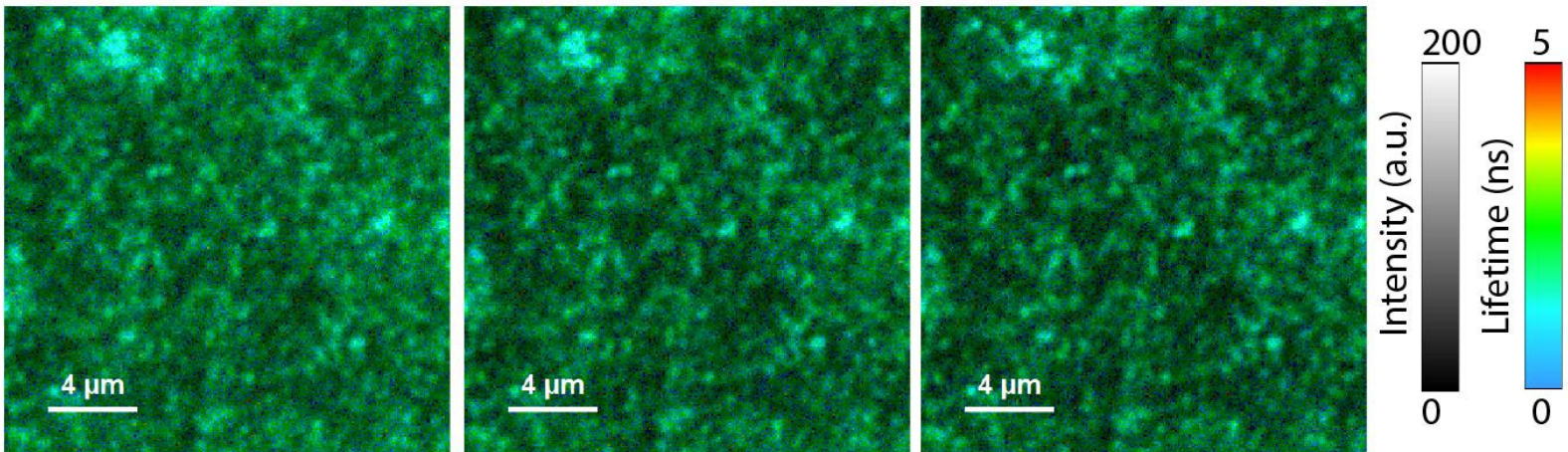

(B) I-phase

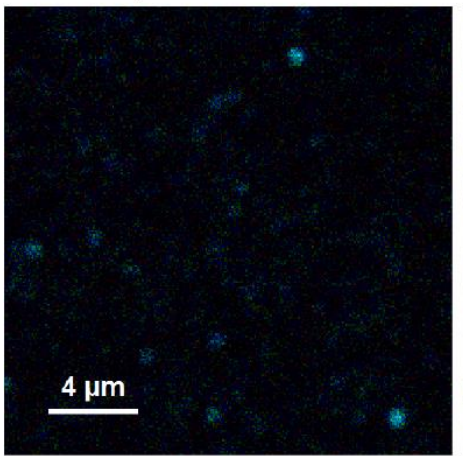

$1_{\text {st }}$ scan

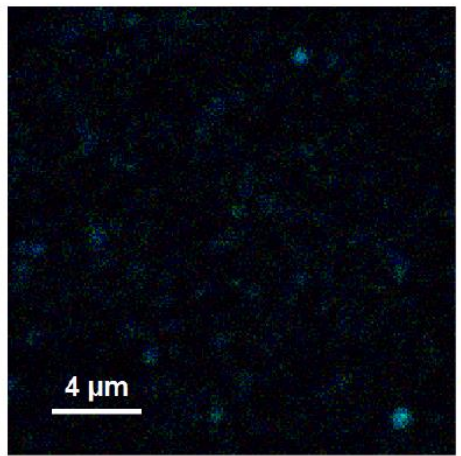

$2_{\text {nd }}$ scan
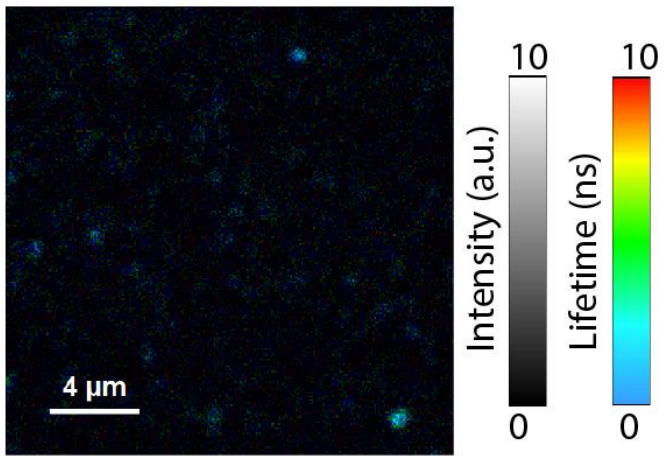

Figure S2. The three consecutive FLIM images of pristine $\mathrm{CsPbIBr}_{2}$ film. PL mapping of (A) Br-phase and (B) I-phase PL confirm that no phase segregation was induced by scanning laser during FLIM measurement. Excitation power density of $500 \mathrm{~mW} / \mathrm{cm}^{2}$ with a dwelling time of $2 \mathrm{~ms}$ at each point.

\section{Supporting Note 1. Discussion on PL intensity and lifetime change during illumination}

During illumination, I-phase PL lifetime is constantly longer than Br-Phase PL, which is the intrinsic properties of I-rich perovskite PL when compared to Br-rich perovskite. The effective lifetimes of both Br-phase and I-phase decrease with illumination time and the PL decay curves exhibit very similar variation tendency which strongly suggests that the variation originates from the same mechanism. Together with the opposite change of PL intensity in Br-phase and I-phase during illumination, that is, decrease in Br-phase and increase in I-phase, the excess local electric field from illumination activated mobile ions is proposed as the dominant mechanism.

On the one hand, illumination can induce defect healing, resulting in increases of both PL intensity and lifetime, which does not match with the decreased PL lifetime. On the other hand, under illumination, halide vacancies and interstitials are generated as defects which can enhance non-radiative recombination and result in decreased PL intensity and lifetime. However, this is also inconsistent with 
the intensity variation of I-phase PL. Therefore, merely discussing defects curing/trapping cannot explain the variation of Br-phase and I-phase PL.

Illumination-induced mobile ions create a local electric field which decreases the PL decay lifetime. Such enhanced non-radiative recombination has similar impacts on both Br-phase and I-phase PL, and PL decay lifetime presents the same decrease tendency under continuous illumination. Meanwhile, the change of PL intensity is due to Bromide ion in lattices substituted by iodine, which leads to the decrease of pre-existing Br-phase PL and the increase of newly emerging I-phase PL intensity.

\section{Supporting Note 2. Ion diffusion model derivation}

Since the thickness of the $\mathrm{CsPIBr}_{2}$ film $(100 \mathrm{~nm})$ is very thin compared to the spatial distribution of postillumination I-phase PL (up to $5 \mu \mathrm{m}$ ), we apply an approximation of 2-dimensional in-plane mobile ion diffusion. The activated mobile ion density in the perovskite film is $\mathrm{C}(\mathrm{r}, \mathrm{t})$, where the parameter $\mathrm{r}$ is the radial distance from the illumination point, and $\mathrm{t}$ is the illumination time.

Upon illumination, mobile ions (halide vacancy) are activated. Therefore, at one specific time $\tau$ during continuous constant illumination, mobile ions density equals to the mobile ion generated rate at the illuminated point $\mathrm{r}=0$ :

$$
C^{\prime}(0, \tau)=\psi * \delta(t-\tau)
$$

Mobile ions then diffuse in the perovskite film (driven mainly by the density gradient), which can be described as:

$$
\frac{\partial C(r, t)}{\partial t}=D \frac{\partial C^{2}(r, t)}{\partial r^{2}}
$$

where $\mathrm{D}$ is the mobile ion diffusion coefficient. For the instantaneous source of mobile ions at $\mathrm{t}=0$, the 2D diffusion function is:

$$
C^{\prime}(r, t)=\frac{\psi}{4 \pi D t} \exp \left(-\frac{r^{2}}{4 D t}\right)
$$

Since we are studying the constant focus-point illumination, where mobile ions (halide vacancy) are continuously activated by illumination. At the illuminated point $r=0$, we assume that mobile ions density equals to the mobile ion generated rate: 


$$
C(0, t)=\psi \text {, }
$$

By integrating the instantaneous source diffusion function in illumination time, we have

$$
C(r, t)=\frac{\psi}{4 \pi D} \Gamma\left(0, \frac{r^{2}}{4 D t}\right)
$$

where $\Gamma(0, k)$ is the incomplete gamma function $\Gamma(0, k)=\int_{k}^{\infty} p^{-1} \exp (-p) \mathrm{d} p$.

In this model, a point source approximation is applied, which is valid when halide vacancies diffusion away from the illuminated point vicinity. Within the radius of the illumination light spot, mobile ions originate mainly from the activation of illumination. Therefore, this model is used to fit the I-phase PL spatial decay out of the illuminated point vicinity whose radius depends on the absolute value of illumination power ( $>$ the threshold for activation). Note that the radius of the focused light spot is generally defined by the waist of the Gaussian beam (1/e $\mathrm{e}^{2}$ of the maximum). The radius of illumination here is intensity-dependent, different from the general radius of the beam. With illumination powers of $200 \mathrm{~mW} / \mathrm{cm}^{2}, 500 \mathrm{~mW} / \mathrm{cm}^{2}$ and $800 \mathrm{~mW} / \mathrm{cm}^{2}$, the thresholds of fitting distance are $250 \mathrm{~nm}, 600 \mathrm{~nm}$ and 1000nm, respectively.

To allow I-phase PL to be observed in the dark region, the mobile ions activated by the focused laser might go through three consecutive processes: (1) ion activation, (2) ion migration and (3) ion substitution/phase segregation. The proposed model utilizes the observed I-phase PL distribution to estimate the ion migration from the illuminated bright spot to the surrounding region. While the model is valid when emission intensity is proportional to the ion density, the fitted diffusion coefficients using this model are "underestimated" because the migrated ions can only be visualized after the completion of the step (3) ion substitution, and migrated ions without inducing phase segregation in the dark regions are not accounted in this model. 